\title{
DESCRIPTION OF A NEW PROPTOMETER
}

\author{
BY \\ J. R. Mutch, M.D., D.O.M.S. \\ SURGERY DEPARTMENT, ABERDEEN UNIVERSITY
}

AlL physicians and surgeons who have had to examine and treat cases of exophthalmic goitre, are aware how difficult it is to state definitely whether or not exophthalmos is present, and how unconvincing a statement " the exophthalmos decreased after operation " or " the exophthalmos varied from day to day " can be, without the support of figures obtained by actual measurement. Up to the present the instrument available, besides being expensive and difficult to use, is in practice too alarming to this class of patient. For some time I have experimented with an instrument which I have found to be very simple to use and not alarming to the patient, and which will indicate, beyond any doubt, whether the proptosis is progressing or regressing.

The first difficulty to overcome was to find a fixed point or points from which the measurements could be obtained, and after a good deal of experimenting the orbital margins above and below the centre of the cornea were chosen.

The instrument consists of a small metal stand with a central plunger, on the upper part of which is a millimetre scale. When the instrument is placed on a flat surface the scale registers zero. To use the instrument the patient's face must be in a horizontal position, so the patient must either be lying down, or sitting in a chair, with the head bent backwards. The operator stands on the left side of the patient irrespective of the eye to be measured. The patient is told to close the eyes, and direct them straight ahead, e.g., towards the ceiling. The highest point of the cornea as seen through the closed upper eyelid is then noted, and the plunger of the instrument allowed to rest upon it. The feet of the

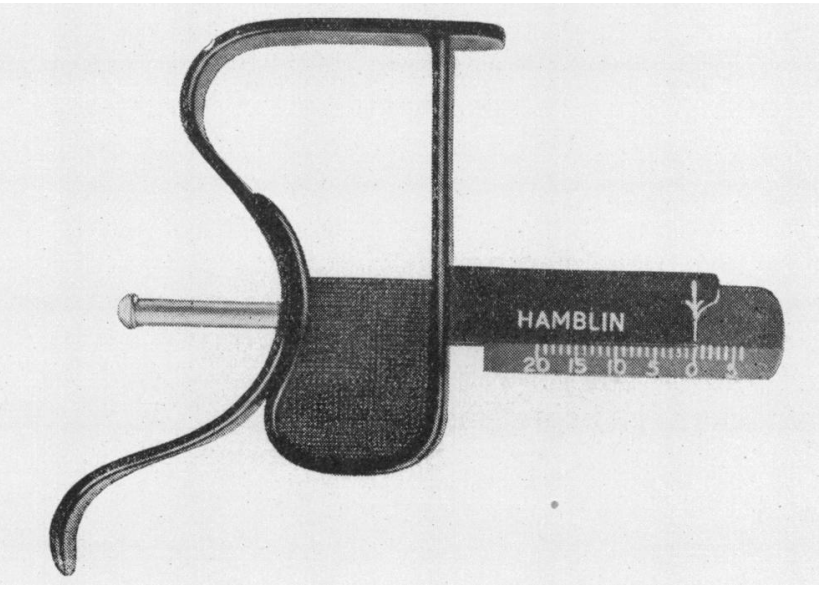


instrument are then allowed to drop until the longer rests on the lower orbital margin, and the shorter on the upper orbital margin. Care has to be taken that the upper projection is resting flat on the bone; in the majority of cases this can be attained only by tilting the instrument a little outwards. Any exophthalmos or enophthalmos present can now be ascertained by reading the scale. Parsons (1938) states " The normal position

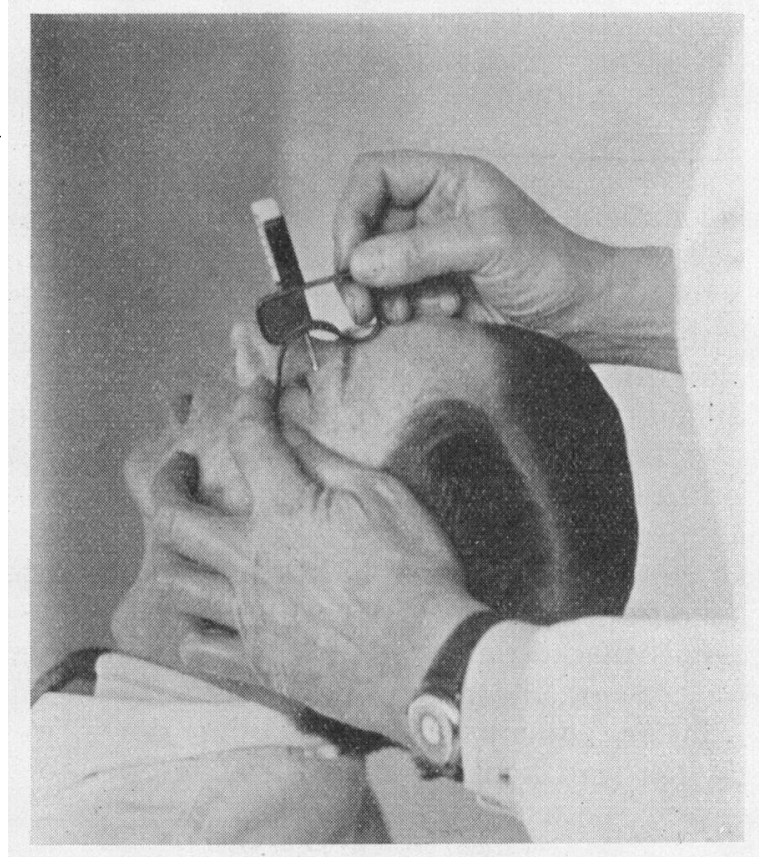

of the eye is such that a straight-edge applied vertically to the middle of the upper and lower margins of the orbit just touches the closed lids over the apex of the cornea."

By measuring a large number of healthy adults, I found that in 90 per cent. the reading was zero. Of the remainder a certain proportion varied from the normal owing to high refractive errors, the variation being due to an alteration in the size of the eyeball, and not to any alteration of position of the eyeball in the socket. With this exception then, the figure registered by the instrument is anatomically a true reading.

It is suggested that + be used to indicate exophthalmos and enophthalmos, and to facilitate quick reading + on the scale is painted red and - black.

The instrument is being manufactured by Messrs. Theodore Hamblin, Ltd., 15, Wigmore Street, London, W.1.

\section{- REFERENCE}

Parsons, J. H.-Diseases of the Eye, 9th Edition, London, 1938. 